\title{
Simulation Analysis in Cognitive Radio Networks with Unreliability and Abandonment
}

\author{
Hamza Nemouchi(D), Mohamed Hedi Zaghouani ${ }^{(凶)}(\mathbb{D}$, and János Sztrik (D) \\ Doctoral School of Informatics, Faculty of Informatics, University of Debrecen, \\ Debrecen, Hungary \\ \{nemouchi.hamza, zaghouani.hedi, sztrik. janos\}@inf .unideb.hu
}

\begin{abstract}
The current paper presents a Cognitive Radio Network with impatient customers and unreliable servers by the help of a finite-source retrial queueing system. We consider two types of customers (primary and secondary) assigned to two interconnected frequency bands. A first frequency band with a priority queue and a second frequency band with an orbit are reserved for Primary Users (PUs) and Secondary Users (SUs), respectively. If the servers are busy, both customers (licensed and unlicensed) enter either the queue or orbit. Before they enter orbit, the secondary customers receive a random retrial time according to the exponential distribution, i.e. the waiting time before the next retry. Unlicensed users (impatient) are obliged to leave the system as soon as their total waiting time exceeds a random maximum waiting time. It should be noted, that the secondary service unit of our system is subject to random breakdowns and repairs. The novelty of this work consists in the investigation of the abandonment and secondary server unreliability impact on various performance measures of the system (Cognitive Radio Network), such as the mean response and waiting time of users, the probability of abandonment of SU, etc. Several figures illustrate the problem in question through simulation.
\end{abstract}

Keywords: Finite source queuing systems $\cdot$ Simulation $\cdot$ Cognitive radio networks $\cdot$ Performance and reliability measures $\cdot$ Non-reliable servers $\cdot$ Impatient customers

\section{Introduction}

Cognitive Radio (CR) is an intelligent technology capable of overcoming the problems of spectrum under-utilization by allowing secondary customers to use the primary channel opportunistically without disrupting primary customer

The research work of János Sztrik is supported by the EFOP-3.6.1-16-2016-00022 project. The project is co-financed by the European Union and the European Social Fund. Mohamed Hedi Zaghouani is supported by the Stipendium Hungaricum Scholarship. 
communications, in order to improve network performance. This intelligent technology is able to modify its transmitter parameters in compliance with the interaction of the environment in which it operates. The main objective of the CRN is to use the unused portions of the primary frequency bands for the benefit of unlicensed customers. Further details can be found in $[1,4,6,9-12,17]$. Several studies and researches, such as $[18,20]$ show that often many parts of the channels are unused in time and space by licensed users (white spaces). Secondary users in these parts of the service unit can detect this non-use and communicate freely without any harmful effects on the primary users. Today there are two types of Cognitive Radio Network. The first type is called (underground) network, where unlicensed users can use the primary channels simultaneously with the licensed users under certain predetermined conditions. The second type is referred to as (overlay) networks, in which the unlicensed users can use the primary service at any time as far as the primary unit is not occupied by licensed users, the authors of $[13,15,19,21]$ have introduced further information. However, the present paper deals with an overlay CR technique by modelling a CRN system containing two finite source subsystems (primary and secondary).

In this queuing system, we take two elements into account. A first subsystem is intended for the jobs of Primary Users (PU) with a finite number of sources. In this part of the system, each source generates a primary call for the PUs after an exponentially distributed time. The latter requests are forwarded to a single server Primary Channel Service (PCS) with a preemptive discipline (FIFO queue) to start the service, assuming that the service time is exponentially distributed. The second component of the model is created for Secondary Users (SU) coming from a finite source and forwarded to Secondary Channel Service (SCS), knowing that the inter-arrival and service times of secondary users are exponentially distributed. The generated primary tasks aim to check the PCS for accessibility. If this service unit is not occupied, the service starts immediately. However, if the PCS is busy with another primary task, this last task will join a First In First Out (FIFO) queue. However, if a second job is being handled in the primary unit, this job is immediately disconnected and should be routed back to the secondary Channel Service. Per the secondary channel's status, the aborted task either restarts the service on its original server (SCS) or joins the retrial queue (Orbit). Besides, the secondary channel also receives low priority requests. If the targeted unit is idle, the service may start immediately. Otherwise, these secondary requests will attempt to join the primary unit. If the primary unit is idle, the secondary requests will have the opportunity to start. If not, they will automatically enter the orbit. From orbit, the postponed requests will retry to receive service after an exponentially distributed random interval. Further details are given in $[6,13,17,21]$. In this study, we assume that impatient customers in orbit whose total waiting time exceeds a random abandonment time which is generally distributed have to leave the system and the second service unit is unreliable which are the novelty of this work. Several studies have examined the Abandonment and/or Unreliability on the basis of different scenarios and systems. At [22] as an example, the authors have pre- 
sented a retrial queueing system with a single server which is subject to random breakdowns and assuming that collisions may occur when a customer arrives at a busy server which forces both jobs to join the orbit. However, to get closer to real-life situations and involving more servers to the system, the authors of [23] examined the abandonment concept on a Cognitive Radio Network by setting a constant value for the maximum waiting time (abandonment time) of secondary users. In an extended work [24], the same authors of the above-mentioned paper assumed that the abandonment time is random, using various distributions to investigate their influence on the main performance measures of such a system. Other probes analysed the abandonment in other types of networks and showed that customers can leave systems from queues, server units while receiving services and while waiting; more details are given in $[7,16]$. However, in the current paper, we assume that impatient users (secondary) are forced to leave the system only from the orbit while waiting. Unreliability of servers was investigated in $[25,26]$, without taking in consideration that customers have the opportunity to leave the system. Several figures will show the effects of the abandonment and unlicensed server unreliability on the performance measures of the system using simulation.

\section{System Model}

Figure 1 demonstrates a finite source queuing system that models the considered cognitive radio network. Our queuing system consists of two not independent, interconnected sub-systems. The first part is allocated to primary requests, with

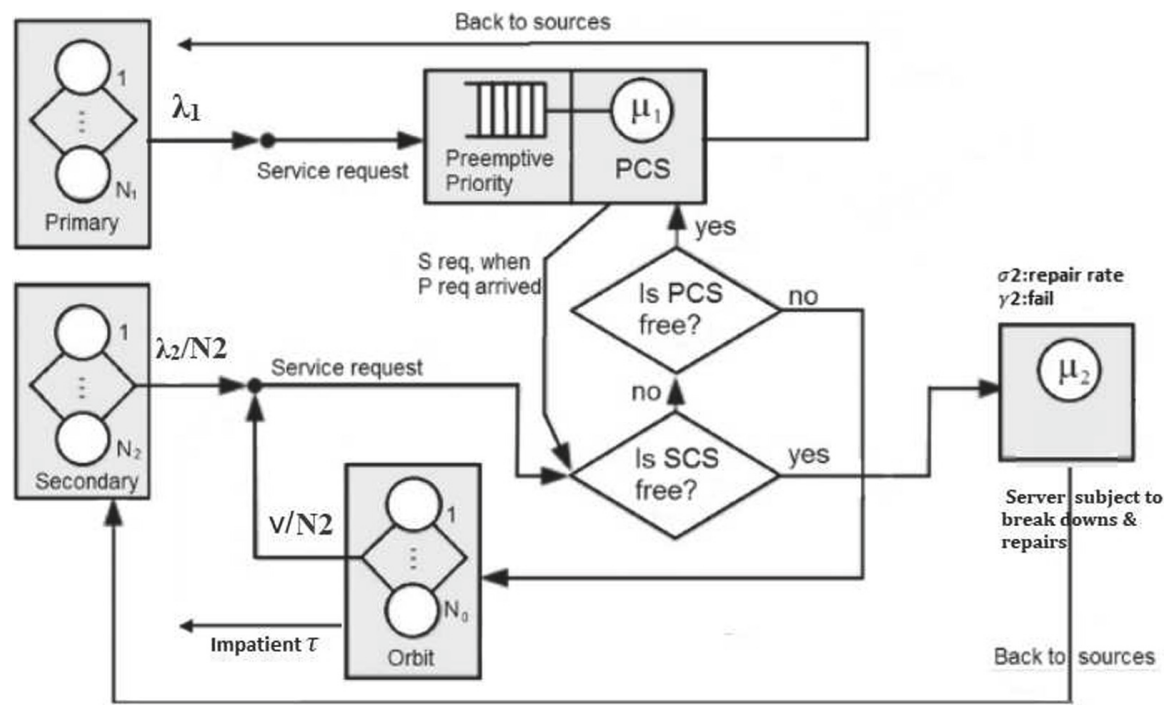

Fig. 1. Finite-source retrial queuing system: Modeling the Cognitive Radio Network with unreliability and abandonment. 
$N_{1}$ the number of sources. These sources will be responsible for generating high priority requests with an inter-request and service times that are exponentially distributed, using parameters $\lambda_{1}$ and $\mu_{1}$, respectively. All the produced requests are directed to a single server unit (PCS) with a preemptive priority over the secondary users. The second subsystem is devoted to the low-priority requests with the number of sources denoted by $N_{2}$, the inter-arrival times and service times in this subsystem are assumed to be exponentially distributed as well, with parameter $\lambda_{2} / N_{2}$ and $\mu_{2}$, respectively. Based on the state of both server (idle or busy), the generated primary packet goes to the primary server (if the server is idle) or joins the FIFO queue (if it is busy with a PU). However, if an unlicensed user occupies the PCS, its service is instantly stopped and will be sent back to the Secondary unit.

Depending on the secondary unit's availability, the aborted task is addressed either to the server or the retrial queue from which reties to get served from the beginning after an exponentially distributed time with parameter $\nu / N_{2}$. On the other hand, requests from SUs are directed to SCS. If it is idle, the service begins. If not, this unlicensed task will sense the PCS. In case of an idle status for PCS, this service may opportunistically join the high priority channel. If the PCS is engaged, the request goes to orbit. It should be noted that Secondary Users in orbit are obliged to leave the system once their total waiting time exceeds a random abandonment time which is generally distributed (Hyper, Hypo, Gamma, Log-normal and Pareto) a rate $\tau$. Random breakdowns during a busy and idle state of the secondary service unit may occur after an exponentially distributed random time with parameters $\gamma_{1}$ and $\gamma_{2}$, respectively. The repair time is also exponentially distributed random variable with parameter $\sigma$.

Assuming that all random variables included in the system are exponentially distributed except the impatience time which is generally distributed random variable, we created a stochastic simulation program written in $\mathrm{C}$ coding language with SimPack [29] libraries. All the numerical results were collected by the validation of the simulation outputs.

\section{Simulation Results}

In this section, several cases are analyzed using a simulation program. The advantage of this later is to make difference between observations during a single run. This difference allows us to investigate the performance measure of two types of cognitive customers (SUs), those who leave the system with successful service and those who abandon the system without a service due to their limited waiting time. Also, the difference between secondary users who left the system with a successful service from the primary service channel and secondary users that leave the system without service after several interruptions at the primary service unit due to the preemptive priority of the primary customers over the secondary ones. In order to estimate the performance measures of these categories, the batch mean value method was used in the simulation. This method is 
a common confidence interval technique used for the analysis of the steady-state simulation output. See for example $[3-5,8]$. By dividing the cognitive users into two categories (Successful and Abandon, we could generate several results. This section of the simulation results is organized following these scenarios:

- Scenario 1: Impatience time of the customers is exponentially distributed.

- Scenario 2: Impatience time is generally distributed with $C_{x}^{2}>1$, using Hyper-Exponential, Gamma, Lognormal and Pareto.

- Scenario 3: Impatience time is generally distributed with $C_{x}^{2}<1$, using Hypo-Exponential, Gamma, Lognormal and Pareto.

In the above scenarios, we suppose that the interrupted secondary service from the PCS due to PUs arrival or from the SCS due to server breakdown will be repeated from the beginning (non-intelligent). Also, the service unit failure will not block the system and the free sources keep generating new calls.

\subsection{Impatience Time is Exponentially Distributed}

In this case, we would like to analyze the main features of the system while all the involved random inter-times are exponentially distributed random variables. The investigation is based on increasing the rate of the impatience times distribution $\tau$.

For the sake of obtaining the following results, the set of parameters values defined in Table 1 must be used.

Table 1. Different impatience rates

\begin{tabular}{l|l|l|l|l|l|l|l|l}
\hline & $N_{2}$ & $\lambda_{2} / N_{2}$ & $\nu / N_{2}$ & $\gamma_{1}$ & $\gamma_{2}$ & $\sigma$ & $\mu_{1}, \mu_{2}$ & $\tau$ \\
\hline Case 1 & 100 & 0.01 & 0.1 & 0.1 & 0.1 & 1 & 1 & 0.000001 \\
\hline Case 2 & 100 & 0.01 & 0.1 & 0.1 & 0.1 & 1 & 1 & 0.0001 \\
\hline Case 3 & 100 & 0.01 & 0.1 & 0.1 & 0.1 & 1 & 1 & 0.001 \\
\hline Case 4 & 100 & 0.01 & 0.1 & 0.1 & 0.1 & 1 & 1 & 0.01 \\
\hline Case 5 & 100 & 0.01 & 0.1 & 0.1 & 0.1 & 1 & 1 & 0.1 \\
\hline Case 6 & 100 & 0.01 & 0.1 & 0.1 & 0.1 & 1 & 1 & 1 \\
\hline
\end{tabular}

For the numerous categories of cognitive users, including successfully served and abandoned ones, we provide accurate estimates in the following. One of the advantages of the simulation is to assist us to perform these measures. We could see some characteristics of our systems in Tables 2, 3, 4, 5 for which the notations are provided in 7 . These results are the estimations mean and variance of the measures based on two scenarios: 
- Scenario A: The arrival traffic of the primary customers is low and small number of sources $\lambda_{1}=0.01, N_{1}=10$.

- Scenario B: The arrival intensity of the primary users is high and large number of sources $\lambda_{1}=0.1, N_{1}=100$.

Table 2. Estimation of the expectations for scenario A

\begin{tabular}{l|l|l|l|l|l|l|l}
\hline & $E(T S)$ & $E(W S)$ & $E(T)$ & $E(W)$ & $E(N S)$ & $E(T A)$ & $P a$ \\
\hline Case 1 & 14.0437 & 13.8001 & 14.04 & 13.8001 & 48.59 & 0.0000 & 0.0000 \\
\hline Case 2 & 14.0525 & 13.8165 & 14.05 & 13.8284 & 44.64 & 15.0001 & 0.001 \\
\hline Case 3 & 13.8333 & 13.5979 & 13.59 & 13.0235 & 38.17 & 15.226 & 0.012 \\
\hline Case 4 & 12.3461 & 12.1107 & 12.48 & 12.27 & 28.33 & 13.5472 & 0.15 \\
\hline Case 5 & 5.5598 & 5.3241 & 6.0853 & 5.9801 & 12.21 & 6.4914 & 0.56 \\
\hline Case 6 & 0.8258 & 0.5908 & 0.9654 & 0.3491 & 5.2454 & 0.9772 & 0.9217 \\
\hline
\end{tabular}

Table 3. Estimation of the variances for scenario A

\begin{tabular}{l|l|l|l|l|l}
\hline & $\operatorname{Var}(T S)$ & $\operatorname{Var}(W S)$ & $\operatorname{Var}(T)$ & $\operatorname{Var}(W)$ & $\operatorname{Var}(T A)$ \\
\hline Case 1 & 197.227 & 190.66 & 197.227 & 197.227 & 0.0000 \\
\hline Case 2 & 197.473 & 190.897 & 197.47 & 190.87 & 185.249 \\
\hline Case 3 & 191.36 & 184.902 & 191.35 & 181.35 & 185.652 \\
\hline Case 4 & 152.42 & 146.67 & 152.48 & 146.66 & 183.52 \\
\hline Case 5 & 30.9119 & 28.3471 & 30.91 & 28.43 & 42.13 \\
\hline Case 6 & 0.6818 & 0.3491 & 0.6820 & 0.3491 & 0.955 \\
\hline
\end{tabular}

Table 2 and 3 determine the values of the expectations and variances of different types of cognitive users, respectively. These results are the outputs of the simulation while $\lambda_{1}=0.01$ and $N_{1}=10$. The rows of the tables define the cases where the impatience rate $\tau$ is increasing. It is clearly seen that the mean and variance values of the response, waiting and arbitrary users are decreasing while the probability of abandonment is increasing. This later increases while an elevation of impatience rate. Unexpectedly, the mean and variance values of the impatient customers are increasing then decreasing during the growth of the abandonment rate. The interpretation of this phenomenon can be as follows: when the impatience rate is very small, the waiting time of the customers is very long, as a result, they rarely leave the system. Therefore, the confidence interval of the expectation for a small set of observations can be very large, thus, the estimation is not accurate.

In Table 4 and 5, the same features as above were treated but in this case the primary traffic more intense supposing $\lambda_{1}=0.1$ and $N 1=100$. The first thing we notice comparing with Tables 2 and 3 is the efficiency of cognitive technology. It 
Table 4. Estimation of the expectations for scenario B

\begin{tabular}{l|l|l|l|l|l|l|l}
\hline & $E(T S)$ & $E(W S)$ & $E(T)$ & $E(W)$ & $E(N S)$ & $E(T A)$ & $P a$ \\
\hline Case 1 & 25.0657 & 24.8343 & 25.0651 & 24.8338 & 57.28 & 29.5803 & 0.000023 \\
\hline Case 2 & 24.9055 & 24.66 & 24.8423 & 24.6119 & 57.28 & 26.5792 & 0.002 \\
\hline Case 3 & 24.2940 & 24.0632 & 24.3561 & 24.1307 & 52.26 & 26.9554 & 0.02 \\
\hline Case 4 & 20.0967 & 19.8643 & 20.4913 & 20.3042 & 30.15 & 22.118 & 0.194 \\
\hline Case 5 & 6.5178 & 6.2909 & 7.2363 & 7.1664 & 6.07 & 7.5563 & 0.61 \\
\hline Case 6 & 0.8242 & 0.6068 & 0.9743 & 0.3682 & 0.9598 & 0.9834 & 0.943 \\
\hline
\end{tabular}

Table 5. Estimation of the variances for scenario B

\begin{tabular}{l|l|l|l|l|l}
\hline & $\operatorname{Var}(T S)$ & $\operatorname{Var}(W S)$ & $\operatorname{Var}(T)$ & $\operatorname{Var}(W)$ & $\operatorname{Var}(T A)$ \\
\hline Case 1 & 628.037 & 616.75 & 628.29 & 616.57 & 726.24 \\
\hline Case 2 & 620.037 & 608.519 & 608.59 & 606.84 & 724.40 \\
\hline Case 3 & 590.201 & 579.04 & 590.2013 & 579.03 & 726.65 \\
\hline Case 4 & 403.8815 & 394.591 & 403.8813 & 394.5913 & 489.2139 \\
\hline Case 5 & 42.4828 & 39.5766 & 42.4828 & 39.4741 & 57.0989 \\
\hline Case 6 & 0.6771 & 0.3682 & 0.6794 & 0.3682 & 0.9671 \\
\hline
\end{tabular}

is shown that when a very small impatience rate $(\tau=0.000001)$, the probability of abandonment is zero in Table 2 , row 1 since the licensed service channel in scenario A is most of the time idle from primary customers. However, besides the larger values of the mean and variance in scenario B. Same explanation as previously for the expectation and variance waiting time of the impatient customers (Table 6).

\subsection{Impatience Time is Generally Distributed with $C_{x}^{2}>1$}

This subsection is Scenario 2 of our investigation, we have analyzed the impact of abandonment time distributions on the main characteristics of the system.

Table 6. Estimation of the variances for scenario A

\begin{tabular}{l|l}
\hline Notation & Definition \\
\hline $\mathrm{E}(\mathrm{TS}), \operatorname{Var}(\mathrm{TS})$ & Mean and variance response time of successful cognitive users \\
\hline $\mathrm{E}(\mathrm{WS}), \operatorname{Var}(\mathrm{WS})$ & Mean and variance waiting time of successful cognitive users \\
\hline $\mathrm{E}(\mathrm{T}), \operatorname{Var}(\mathrm{T})$ & Mean and variance response time of arbitrary cognitive users \\
\hline $\mathrm{E}(\mathrm{W}), \operatorname{Var}(\mathrm{W})$ & Mean and variance waiting time of arbitrary cognitive users \\
\hline $\mathrm{E}(\mathrm{NS})$ & Mean number of secondary customers in the system \\
\hline $\mathrm{E}(\mathrm{TA}), \operatorname{Var}(\mathrm{TA})$ & Mean and variance waiting time of impatient customers \\
\hline $\mathrm{Pa}$ & Probability of abandonment \\
\hline
\end{tabular}


In this scenario, we consider that the impatience time is hyper-exponentially, gamma, Pareto and, lognormally distributed random variable with the same mean and same variances. In order to calculate the shape, rate, and scale of these distributions, see [28]. Table 7 defines the numerical values of distribution parameters.

The inter-events times are generated using several methods of random numbers generator. These methods require input parameters which in our case are the rates of the distributions. The numerical values of these parameters are defined in Table 8 .

Table 7. Parameters of the distributions

\begin{tabular}{l|l|l|l|l}
\hline Distribution & Gamma & Hyper-exponential & Pareto & Lognormal \\
\hline Parameters & $\alpha=0.390625$ & $p=0.33098$ & $\alpha=2.1892$ & $m=5.5797$ \\
& $\beta=0.0007813$ & $\begin{array}{l}\lambda_{1}=0.00132 \\
\lambda_{2}=0.00268\end{array}$ & $k=270.5630$ & $\sigma=1.12684$ \\
\hline Mean & 500 & & \\
\hline Variance & 640000 & \multicolumn{5}{|l}{} & \\
\hline$C_{x}^{2}$ & 2.56 & & \\
\hline
\end{tabular}

Table 8. Numerical values of the parameters

\begin{tabular}{l|l|l|l|l|l|l|l|l|l|l}
\hline$N_{1}$ & $N_{2}$ & $\lambda_{1}$ & $\lambda_{2} / N_{2}$ & $\mu_{1}$ & $\mu_{2}$ & $\nu / N_{2}$ & $\tau$ & $\gamma_{1}$ & $\gamma_{2}$ & $\sigma_{2}$ \\
\hline 10 & 100 & 0.01 & x - axis & 4 & 4 & 0.01 & 0.002 & 0.01 & 0.01 & 1 \\
\hline
\end{tabular}

\section{Comments}

In all the obtained results, it should be noticed that beside the abandonment time, all the inter-event times in the system are supposed to be exponentially distributed random variables.

Figure 2 illustrates the impact of the impatience time distributions on the mean sojourn time of the cognitive users that leave the system after a successful service while increasing the secondary arrival intensity. The Pareto distribution gives the smallest value of the mean response while the gamma distribution gives the greatest value. This relative difference could be explained by the help of the density function of each distribution. Also, if we read the papers that investigated a single server finite-source retrial queueing system with the abandonment of customers, we notice that the relative difference of the mean values between the lognormal and the hyper-exponential distributions is smaller then the one 


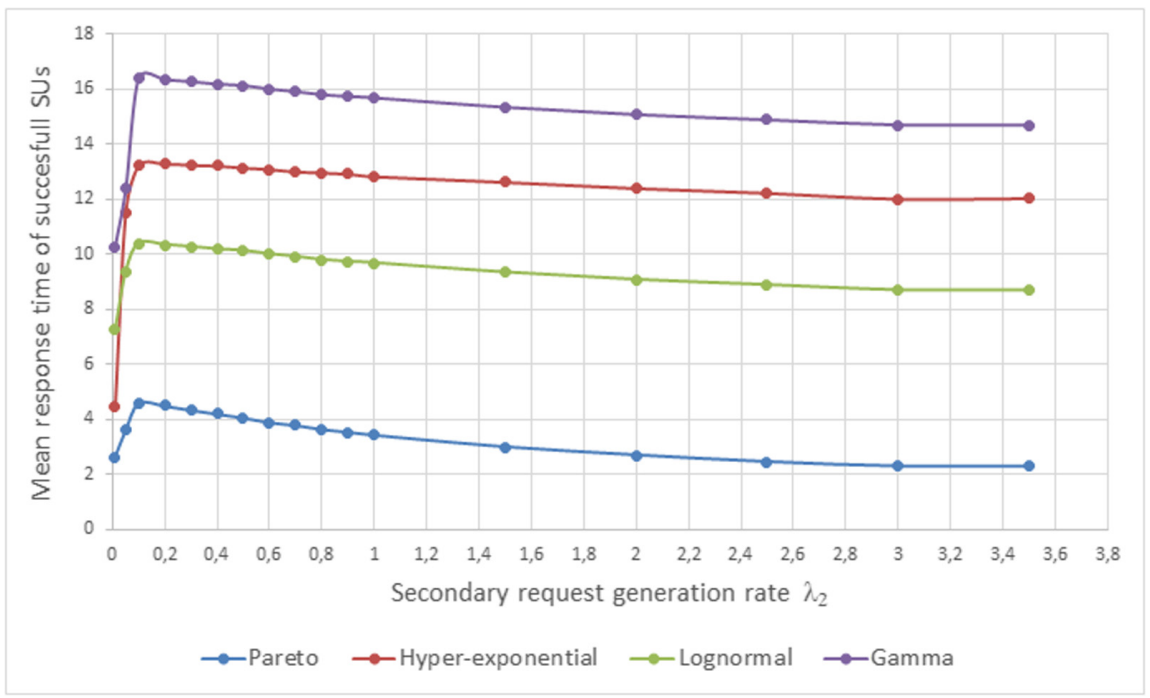

Fig. 2. The impact of the impatience time distributions on the mean sojourn time of successful cognitive users vs secondary request generation rate

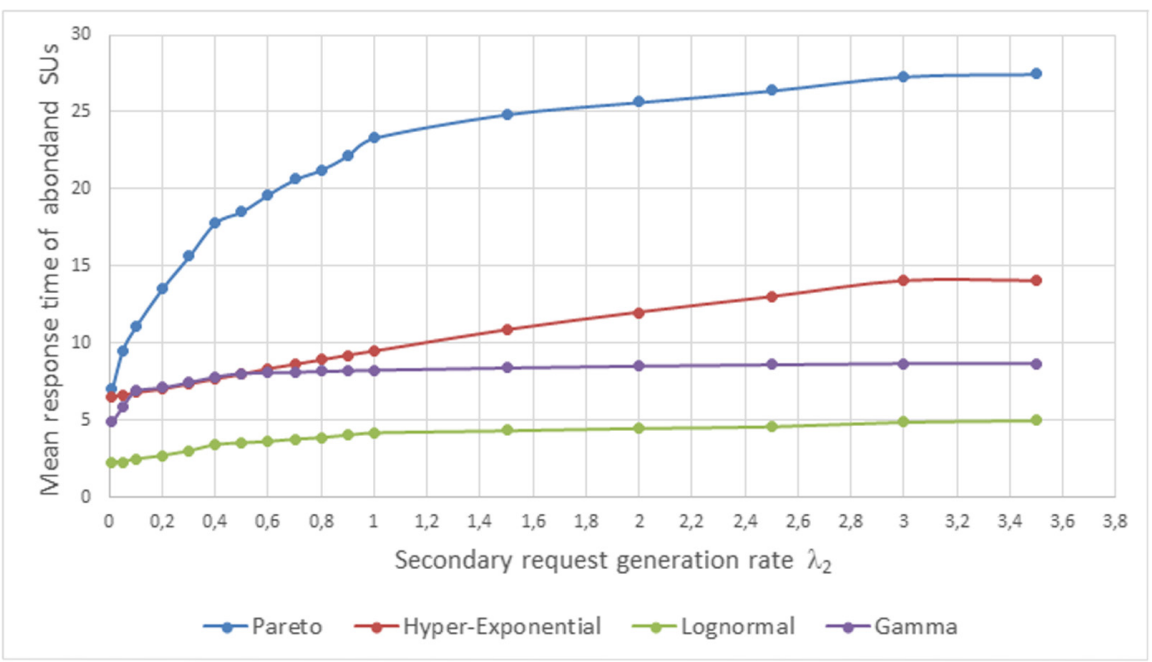

Fig. 3. The impact of the impatience time distributions on the mean sojourn time of impatient cognitive users vs secondary request generation rate 


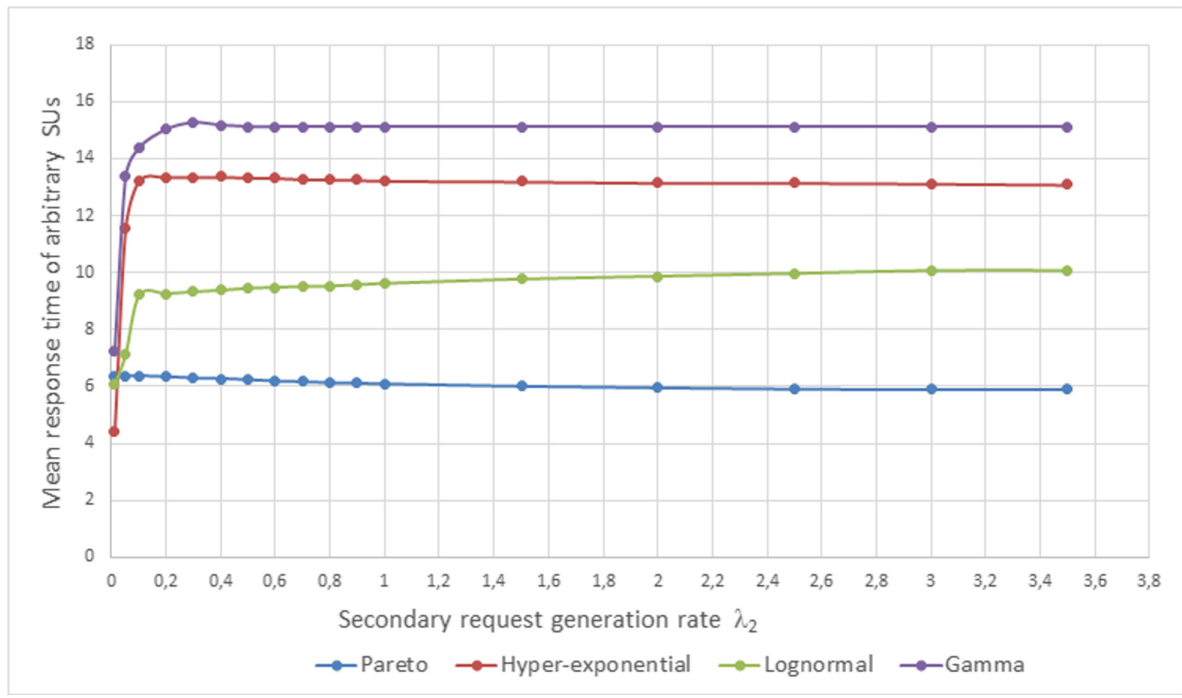

Fig. 4. The impact of the impatience time distributions on the mean sojourn time of arbitrary cognitive users vs secondary request generation rate

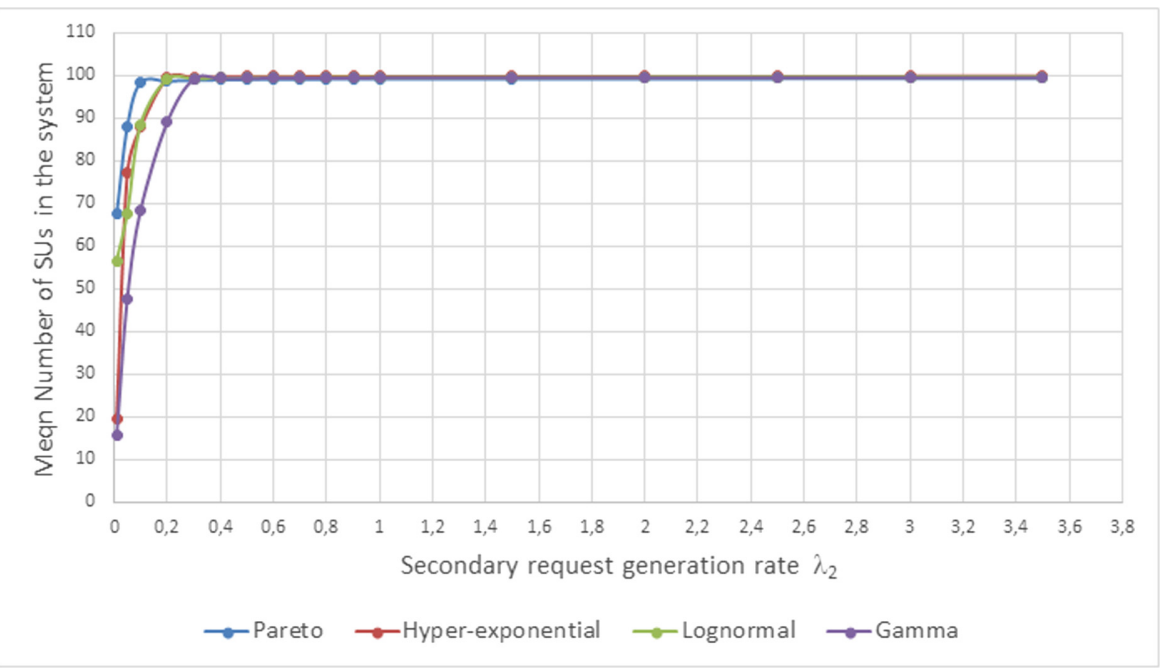

Fig. 5. The mean number of cognitive users in the system vs secondary arrival intensity 
obtained in Fig. 1. This difference is obviously due to the introduction of a second server and a cognitive characteristic to the secondary customers which make the system more complex. However, the maximum property of the mean response time that was noticed in [27] is obtained.

Figure 3 shows the effect of the abandonment time distributions on the mean response time of the secondary customers that leave the system without getting served. The result shows that while increasing the request generation intensity, the value of the mean increases. The aim of making a difference between successful and impatient customer is obtained in this figure, we see that the Pareto distribution gives the greatest value of the mean, while for this feature, the lognormal distribution shows the smallest value.

With the help of the law of total expectation, it is easy to calculate the mean of arbitrary users. Figure 4 illustrates the effect of the impatience time distribution on the mean response time of an arbitrary user while the secondary request generation rate is increasing. The mean value of arbitrary users depends on the probability of success and the probability of abandonment. As the gamma distribution gives a small value of the impatience time, it involves a high probability of abandonment, thus, the value of the mean response time of arbitrary users is the greatest as shown in the figure.

Figure 5 illustrates the mean number of secondary customers in the function of the second generation request rate while the impatience time is generally distributed. The effect of the distributions can be seen when the system is low loaded. When the arrival intensity increases, the mean number of cognitive customers increases, and the distributions have no more impact on its value.

\subsection{Impatience Time is Generally Distributed with $C_{x}^{2}<1$}

In Scenario 3 of our investigation, we set the parameters of the used distribution in a way their squared coefficient of variation becomes less than one. For this case, we use the two phases of hypo-exponential distribution. The aim is to analyze their effects on the main performance measures of the system. The new set of the distribution parameters and the numerical values of the simulation input parameters are shown in Table 9 and Table 10, respectively.

Table 9. Parameters of the distributions

\begin{tabular}{l|l|l|l|l}
\hline Distribution & Gamma & Hypo-exponential & Pareto & Lognormal \\
\hline Parameters & $\alpha=1.47059$ & $\lambda_{1}=0.01$ & $\alpha=2.5718$ & $m=5.9552$ \\
& $\beta=0.002941$ & $\lambda_{2}=0.0025$ & $k=305.5844$ & $\sigma=0.72027$ \\
\hline Mean & 500 & & \\
\hline Variance & 170000 & & \\
\hline$C_{x}^{2}$ & 0.68 & & & \\
\hline
\end{tabular}




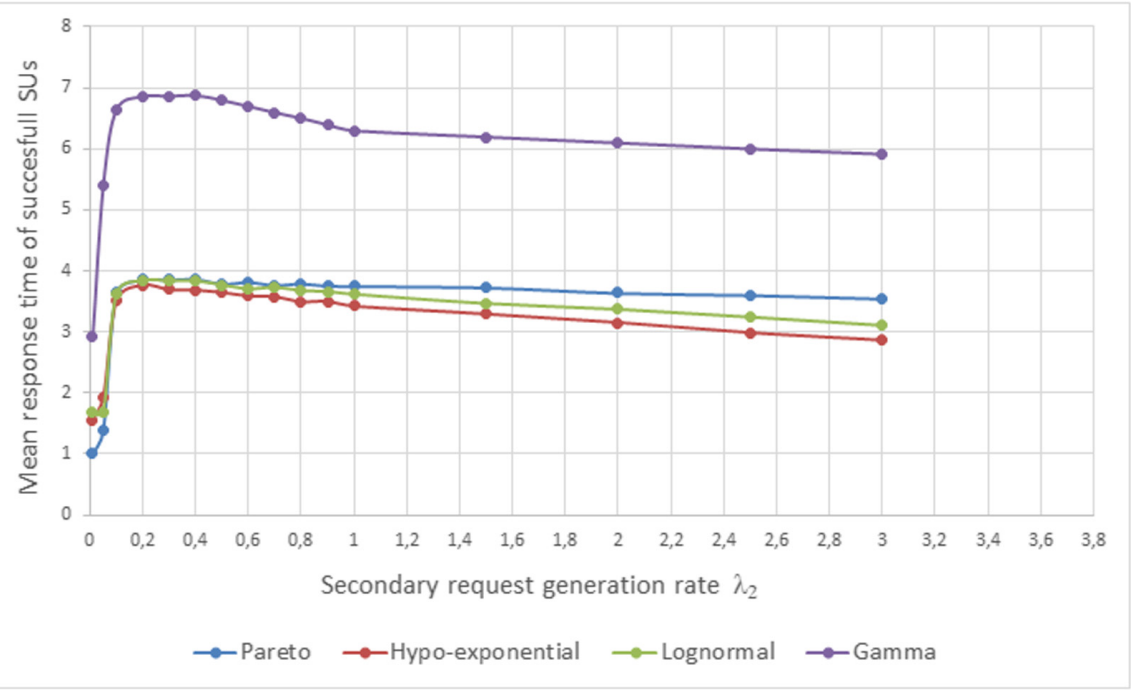

Fig. 6. The effect of the impatience time distributions on the mean sojourn time of successful cognitive users vs secondary request generation rate

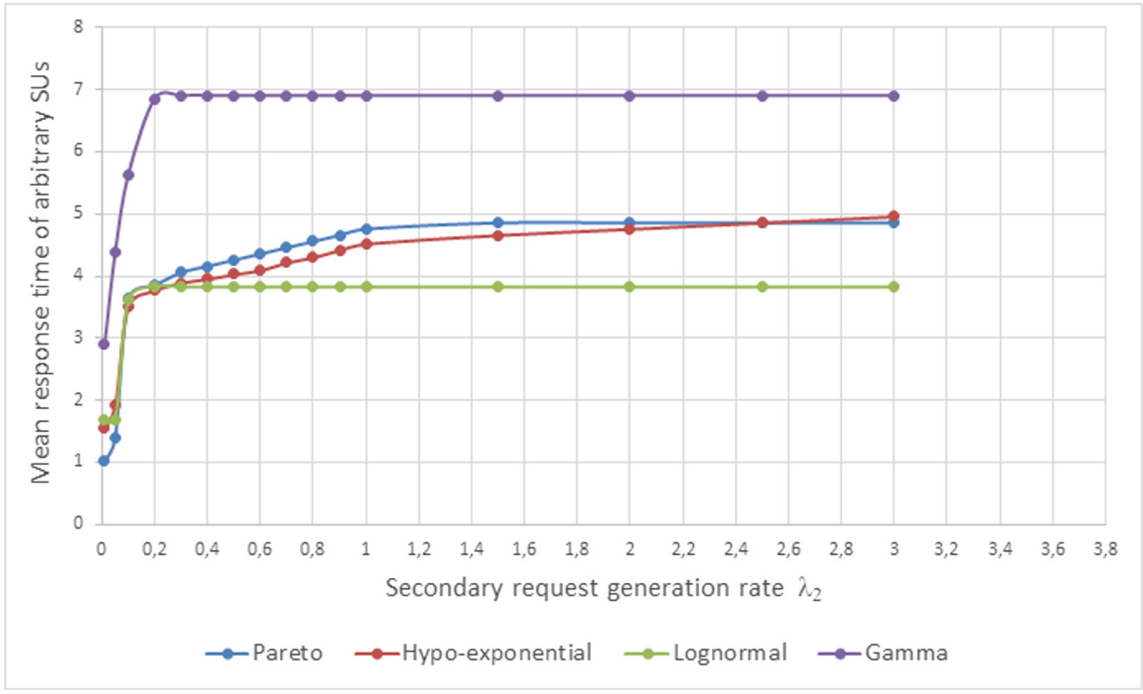

Fig. 7. The effect of the impatience time distributions on the mean sojourn time of arbitrary cognitive users vs secondary request generation rate

Table 10. Numerical values of the parameters

\begin{tabular}{l|l|l|l|l|l|l|l|l|l|l}
\hline$N_{1}$ & $N_{2}$ & $\lambda_{1}$ & $\lambda_{2} / N_{2}$ & $\mu_{1}$ & $\mu_{2}$ & $\nu / N_{2}$ & $\tau$ & $\gamma_{1}$ & $\gamma_{2}$ & $\sigma_{2}$ \\
\hline 10 & 100 & 0.01 & x - axis & 4 & 4 & 0.01 & 0.002 & 0.01 & 0.01 & 1 \\
\hline
\end{tabular}




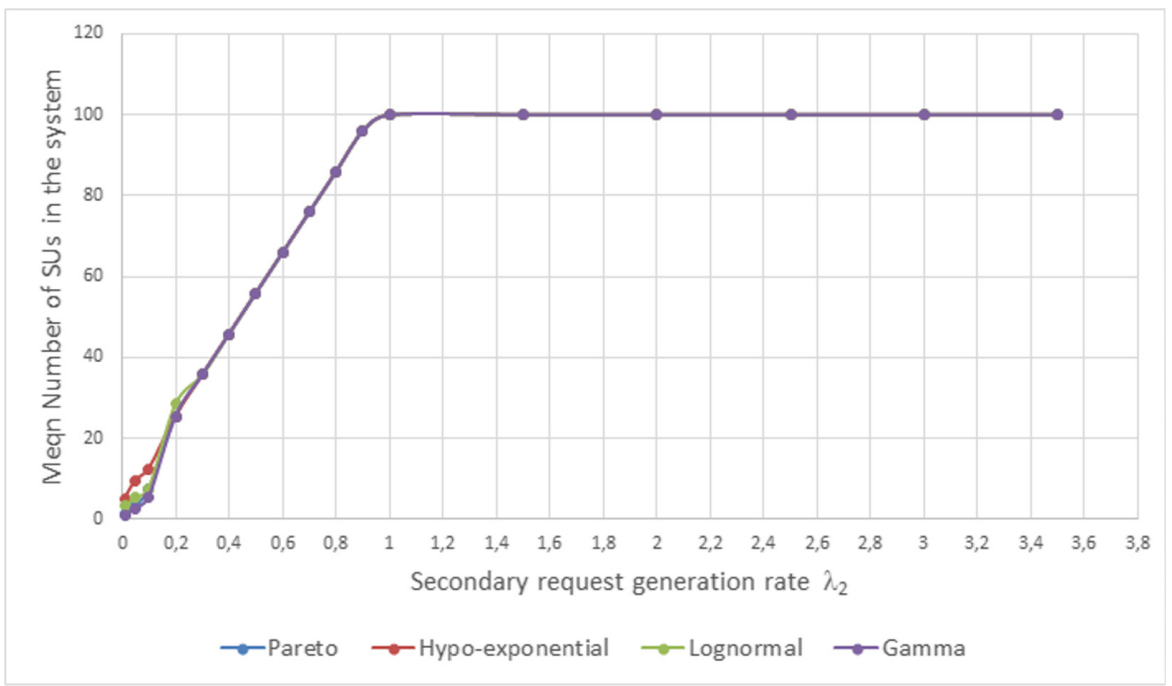

Fig. 8. The mean number of cognitive users in the system vs secondary arrival intensity

\section{Comments}

In terms of arrival intensity, Fig. 6 and Fig. 7 display the mean residence time of successful and arbitrary customers, respectively. The same pattern can be seen in the analysis of the outputs as in the previous corresponding figures, but variations can also be seen, especially in case of the gamma distribution. These statistics also indicate that, relative to the previous parameter setting, successful and arbitrary users spend less time on the system on average.

Lastly, Fig. 8 illustrates the mean number of secondary customers in the system in the function of the secondary arrival intensity. Among a highly loaded system, the figure shows no impact of the distribution on the mean number of customers, but as a low loaded system, a slight difference can be investigated. Also, relative to the previous set of parameters with the corresponding figure, there are fewer customers in the system when the squared coefficient of variation of the distributions is less than one.

\section{Conclusion}

In this paper, a finite-source cognitive radio network was modelled with the help of a retrial queueing system with impatient customers and a secondary server non-reliable. The results have demonstrated the impact of the abandonment time distribution on the mean and variance of the main characteristic of such a complex system. The efficiency of the primary service channel and of the cognitive property at the secondary users was also demonstrated. Using simulation, we succeeded to separate the secondary customers into three categories (impatient/successful/arbitrary) and analyze their performances separately. Based on 
this work, our perspective for the future is to introduce intelligent cognitive users which mean that a secondary user will continue the interrupted (due to primary arrival or server breakdown) service and he will not repeat it from the beginning and demonstrate the distribution of the customers (primary and secondary) in such a system while both subsystems are non-independent. Also, with the help of simulation, we can investigate separately the customers that leave the system successfully from the primary service channel and their mean interrupted service time.

\section{References}

1. Akyildiz, I.F., Lee, W.Y., Vuran, M.C., Mohanty, S.: Next generation/dynamic spectrum access/cognitive radio wireless networks: a survey. Comput. Netw. 50(13), 2127-2159 (2006)

2. Almási, B., Bérczes, T., Kuki, A., Sztrik, J., Wang, J.: Performance modeling of finite-source cognitive radio networks. Acta Cybernetica 22(3), 617-631 (2016)

3. Carlstein, E., et al.: The use of subseries values for estimating the variance of a general statistic from a stationary sequence. Ann. Stat. 14(3), 1171-1179 (1986)

4. Chen, E.J., Kelton, W.D.: A procedure for generating batch-means confidence intervals for simulation. Checking independence and normality. Simulation 83(10), 683-694 (2007)

5. Fishman, G.S., Yarberry, L.S.: An implementation of the batch means method. FORMS J. Comput. 9(3), 296-310 (1997)

6. Gunawardena, S., Zhuang, W.: Modeling and Analysis of Voice and Data in Cognitive Radio Networks. Springer, Heidelberg (2014)

7. He, Q.M., Zhang, H., Ye, Q.: An M/PH/K queue with constant impatient time. Math. Methods Oper. Res. 87(1), 139-168 (2018)

8. Law, A.M., Kelton, W.D.: Simulation Modeling and Analysis, vol. 3. McGraw-Hill, New York (2000)

9. Nemouchi, H., Sztrik, J.: Performance simulation of finite-source cognitive radio networks with servers subjects to breakdowns and repairs. J. Math. Sci. 237(5), 702-711 (2019)

10. Nemouchi, H., Sztrik, J.: Performance evaluation of finite-source cognitive radio networks with collision using simulation. In: 8th IEEE International Conference on Cognitive Infocommunications (CogInfoCom), pp. 000127-000131. IEEE (2017)

11. Nemouchi, H., Sztrik, J.: Performance evaluation of finite-source cognitive radio networks with non-reliable services using simulation. Annales Mathematicae et Informaticae, vol. 49, pp. 109-122. Eszterházy Károly University Institute of Mathematics and Informatics (2018)

12. Nemouchi, H., Sztrik, J.: Performance simulation of non-reliable servers in finitesource cognitive radio networks with collision. In: Dudin, A., Nazarov, A., Kirpichnikov, A. (eds.) ITMM 2017. CCIS, vol. 800, pp. 194-203. Springer, Cham (2017). https://doi.org/10.1007/978-3-319-68069-9_16

13. Paluncic, F., Alfa, A.S., Maharaj, B.T., Tsimba, H.M.: Queueing models for cognitive radio networks: a survey. IEEE Access 6, 50801-50823 (2018)

14. Sztrik, J., Almási, B., Roszik, J.: Heterogeneous finite-source retrial queues with server subject to breakdowns and repairs. J. Math. Sci. 132(5), 677-685 (2006)

15. Van Do, T., Do, N.H., Horváth, Á., Wang, J.: Modelling opportunistic spectrum renting in mobile cellular networks. J. Netw. Comput. Appl. 52, 129-138 (2015) 
16. Wang, J., Abouee Mehrizi, H., Baron, O., Berman, O.: Staffing Tandem Queues with Impatient Customers-Application in Financial Service Operations. Rotman School of Management Working Paper 3116815 (2018)

17. Wang, L., Wang, C., Adachi, F.: Load-balancing spectrum decision for cognitive radio networks. IEEE J. Sel. Areas Commun. 29(4), 757-769 (2011)

18. Weiss, T.A., Jondral, F.K.: Spectrum pooling: an innovative strategy for the enhancement of spectrum efficiency. IEEE Commun. Mag. 42(3), S8-14 (2004)

19. Wong, E.W., Foh, C.H.: Analysis of cognitive radio spectrum access with finite user population. IEEE Commun. Lett. 13(5), 294-296 (2009)

20. Zaghouani, M.H., Sztrik, J., Uka, A.: Simulation of the performance of Cognitive Radio Networks with unreliable servers. Annales Mathematicae et Informaticae (2020)

21. Zekavat, S.A., Li, X.: User-central wireless system: ultimate dynamic channel allocation. In: First IEEE International Symposium on New Frontiers in Dynamic Spectrum Access Networks. DySPAN, pp. 82-87. IEEE (2005)

22. Kuki, A., Bérczes, T., Tóth, Á., Sztrik, J.: Numerical analysis of finite source Markov retrial system with non-reliable server, collision, and impatient customers. Annales Mathematicae et Informaticae 51, 53-63 (2020)

23. Zaghouani, M.H., Sztrik, J.: Performance evaluation of finite-source Cognitive Radio Networks with impatient customers. Annales Mathematicae et Informaticae 51, 89-99 (2020)

24. Zaghouani, M.H., Sztrik, J.: Performance simulation of finite-source Cognitive Radio Networks with impatient calls in the orbit, ISSPSM (2020)

25. Sztik, J., Zaghouani, M.H., Uka, A.: Reliability analysis of cognitive radio networks. In: 18th International Conference named after A.F. Terpugov. Information Technologies and Mathematical Modelling ITMM-2019, Saratov, Russia (2019)

26. Nemouchi, H., Zaghouani, M.H., Sztrik, J.: The impact of servers reliability on the characteristics of cognitive radio systems. In: The 1st Conference on Information Technology and Data Science CITDS (2020)

27. Nazarov, A., Sztrik, J., Kvach, A.: A survey of recent results in finite-source retrial queues with collisions. In: Dudin, A., Nazarov, A., Moiseev, A. (eds.) ITMM/WRQ -2018. CCIS, vol. 912, pp. 1-15. Springer, Cham (2018). https://doi.org/10.1007/ 978-3-319-97595-5_1

28. Kuki, A., Berczes, T., Sztrik, J., Toth A.: Reliability analysis of a two-way communication system with searching for customers. In: Proceedings of The International Conference on Information and Digital Technologies, pp. 260-265. IEEE (2019)

29. Fishwick, P.A.: Getting started with simulation programming in $\mathrm{C}$ and $\mathrm{C}++$. In: Winter Simulation Conference, pp. 154-162 (1992) 